\title{
ON THE CONCEPT OF ENDOGENOUS VOLATILITY
}

\author{
Orlando Gomes ${ }^{1}$ \\ Lisbon Higher Institute of Accountancy and Administration (ISCAL/IPL) \\ and Business Research Unit (BRU/UNIDE/ISCTE).
}

- March 2011 -

${ }^{1}$ Address: Lisbon Higher Institute of Accountancy and Administration (ISCAL/IPL), Avenida Miguel Bombarda 20, 1069-035 Lisbon, Portugal. E-mail: omgomes@iscal.ipl.pt 


\begin{abstract}
Most financial and economic time-series display a strong volatility around their trends. The difficulty in explaining this volatility has led economists to interpret it as exogenous, i.e., as the result of forces that lie outside the scope of the assumed economic relations. Consequently, it becomes hard or impossible to formulate short-run forecasts on asset prices or on values of macroeconomic variables. However, many random looking economic and financial series may, in fact, be subject to deterministic irregular behavior, which can be measured and modelled. We address the notion of endogenous volatility and exemplify the concept with a simple business-cycles model.
\end{abstract}

Keywords: Endogenous volatility, Volatility clustering, Nonlinear dynamics, Chartists and fundamentalists, Periodicity and chaos, Business cycles. 


\section{INTRODUCTION}

The forces that shape the evolution of a price or the motion in time of a given real variable are so many that economists are often faced with a sentiment of frustration. No matter how much we know about the way markets are organized, or about the relative weight of each market participant, or even about the intrinsic complexity governing the relations between agents, we will never be able to accurately understand how economic and financial variables will evolve in the near future. This implies that there is a random component underlying this evolution. The future always brings unexpected events, introducing uncertainty into the economic environment and an impossibility of exactly knowing what the next movement of, e.g., an asset price or an interest rate, will be.

A fundamental question is whether the observed volatility is entirely associated to unpredictable events or if there is a more or less significant part of this volatility that is endogenous, emerging from the type of relation that is established between the relevant economic or financial variables. The answer to this question has huge practical implications - endogenous volatility will correspond to the predictable component of the observed fluctuations; if we can discern this, we will be able to isolate a smaller component of true unpredictability and, in this way, mitigate the uncertainty associated with the time trajectory of the variable(s) under consideration.

The observation of the behavior through time of some economic aggregates provides an indication that, effectively, some of the displayed volatility is endogenous. For instance, Mandelbrot (1963) has identified the presence of 'volatility clustering' in the evolution of prices. Under volatility clustering, periods of large volatility alternate with periods of small changes in prices of commodities and assets. The existence of this type of phenomena was pervasively recognized in the academic profession and it begun to be modelled resorting to statistical models of the ARCH type [Engle (1982), Bollerslev (1986)]. These models allow to describe the data but do not explain why volatility clustering is, in fact, observed in many financial and economic time series. The referred phenomenon must be associated with some kind of endogeneity, in the sense that if the volatility was purely random, it would not display any type of regularity, as the one just described.

As Adrangi et al. (2010) refer, many random looking time series may contain deterministic fluctuations and the state of the scientific knowledge allows, at the present, to conduct some analyses in order to distinguish what is endogenous from what is purely random or 'noise'. Unfortunately, many of the undertaken studies so far are not completely conclusive; we will get back to this idea in the beginning of section 3 .

We will be concerned with endogenous volatility essentially at two levels: policy implications and possible theoretical approaches. If we accept the intuition that observed fluctuations are, at least partially, endogenous, we can open the door to short-run predictability. A stochastic / random time series is completely unpredictable; if the fluctuations are deterministic, on the other hand, even when they are irregular the possibility to forecast future values with accuracy exists if one is able to fully understand the law 
of motion governing the relation between endogenous variables. Deterministic cycles may be periodic, of any possible periodicity, or completely a-periodic, i.e., we may have endogenous irregular fluctuations which can be associated with the notion of chaos. Chaotic time series, that we will address with some detail in section 2, are characterized by being deterministic (and, thus, full predictability exists) but also by being subject to sensitive dependence on initial conditions (SDIC), what means that even if we are in the possession of the actual law of motion governing the economic or financial relation, we can radically fail in providing good forecasts on future values of the series, namely if an error occurs in understanding the initial state of the system; even the slightest difference in initial conditions leads to a complete divergence of the considered orbits.

From the theoretical perspective, we will be concerned with emphasizing the idea that a simple nonlinear dynamic relation is capable of generating endogenous cycles. The only pre-requisite for these cycles to emerge is, in fact, the lack of linearity. Noticing that the reality is complex and that most of the relations in the financial and economic realms are necessarily nonlinear, we infer that endogenous volatility is not difficult to explain from a theoretical point of view. This is the strong idea that the paper explores, first by surveying theory and applications in this field of study and, in a second stage, by illustrating the presence of endogenous cycles in a macroeconomic business cycles model.

The remainder of the paper is organized as follows. Section 2 reviews the most meaningful notions on nonlinear dynamics. Section 3 describes some of the recent applications on economics and finance. In section 4, an illustration is explored; this illustration relates to a business cycles macro model, relatively to which we can address the dynamics of the inflation rate. Section 5 concludes.

\section{THE THEORY OF NONLINEAR DYNAMICS}

Economic relations are typically addressed in a multi-period framework. What has happened in the past has an impact on today's economic activity and current events shape future time paths. Moreover, past expectations on today's outcomes and current expectations about future outcomes are, most of the times, present in this dynamic interpretation of the observed reality. The dynamics can be formally addressed through models involving differential equations (in continuous time) or difference equations (in discrete time). These equations reflect the kind of relations one expects to exist between economic variables; most of the times these are not just $a d-h o c$ relations but the outcome of the optimizing behavior of rational agents.

If the referred relations take a nonlinear form, the dynamic process characterizing the evolution in time of the assumed variables may depart from the trivial results of pure convergence to a fixed-point steady-state (stability) or pure divergence from such point (instability). Cycles of any periodicity or even completely a-periodic motion might arise; in this case, we will be in the presence of bounded instability or endogenous volatility: there will be a perpetual fluctuation around the steady-state point without ever occurring 
a complete convergence or a complete divergence relatively to that point.

As referred in the introduction, the discovery of fluctuations determined by the type of connection between variables has significant impact over the way we understand the evolution of financial and economic time series - irregular behavior is not necessarily synonymous of stochastic behavior and we can find some type of predictability in a series with apparent erratic behavior.

In this section, we address the most significant notions and results on deterministic nonlinear dynamics. Our intention is not to be thorough in the presentation of the theory but rather to highlight the most important tools and intuition at this level. A more detailed presentation of concepts and mechanisms can be found in Medio and Lines (2001), Lines (2005), Barnett et al. (2006), Gomes (2006) and Grandmont (2008).

Our starting point is a difference equation defined in some $m$ dimension (we shall proceed the presentation assuming that time is discrete):

$$
X_{t+1}=G\left(X_{t}\right), \quad X_{0} \text { given }
$$

Function $G$ is a map from an open subset $U$ of $\mathbb{R}^{m}$ into $\mathbb{R}^{m}$, i.e., $G: U \subset \mathbb{R}^{m} \rightarrow \mathbb{R}^{m}$, and $X$ is a vector with $m$ variables $x^{i}, i=1,2, \ldots, m$. The evolution of the set of variables $x$ in time will depend on the particular shape of function $G$.

If $G$ is linear, only two outcomes are possible: the elements of $X$ converge from $X_{0}$ towards a fixed-point $X^{*}$ or, alternatively, they will (at least some of them) diverge from $X_{0}$ relatively to $X^{*}$. Let $G\left(X_{t}\right)=A+B X_{t}$, with $A$ a vector of parameters of length $m$ and $B$ a $m \times m$ square matrix also of parameters. Defining the steady-state, balanced growth path or long-term equilibrium as the point in which the system remains at $X_{t+1}=X_{t}:=X^{*}$, the system's steady-state will be $X^{*}=(I-B)^{-1} A$ with $I$ a $m \times m$ identity matrix. Transitional dynamics (the behavior of the system from $t=0$ to $t \rightarrow \infty$ ) will be determined by the properties of matrix $B$; more accurately, the dynamics will depend on the values assumed by the $m$ eigenvalues of the matrix. The number of eigenvalues for which $\left|\lambda_{i}\right|<1$, i.e. the number of eigenvalues inside the unit circle, determine the dimension of the stable arm; the number of eigenvalues such that $\left|\lambda_{i}\right|>1$, i.e. the number of eigenvalues that fall outside the unit circle, correspond to the unstable dimension of the system. For instance, a three dimensional system with two eigenvalues lower than 1 in absolute value has a stability dimension of order 2 .

In contrast, when nonlinearities are present, we will possibly encounter long-term outcomes that differ from the simple convergence or divergence behavior. A nonlinear system can be linearized in the vicinity of the steady-state and addressed as explained above. Such a procedure may be helpful in order to understand what occurs in a local perspective, i.e. when the initial locus of the system is located nearby the long-term fixed-point result. However, such an approach may hide global dynamics involving much more sophisticated intertemporal behavior. Typically, the local analysis of a nonlinear system is able to separate a region of stability, in the space of the system's parameters, from a 
region of divergence relatively to the fixed-point. The frontier between these two regions is a bifurcation line. The consequences of passing through this bifurcation line become clear once we look at global dynamics: as we abandon the stability area, the bifurcation may trigger the formation of low-periodicity cycles, that may degenerate in more complex long-run outcomes before plain instability sets in. This eventual process of formation of irregular cyclical behavior is the outcome of varying the values of the key parameters of the model.

In a systematic form, we present quick and straightforward definitions of the several types of long-term outcomes we can find when analyzing nonlinear maps (i.e., nonlinear systems in discrete time):

1. Fixed-point: $X^{*}$ is a fixed-point of $X$ if $X^{*}=G\left(X^{*}\right)$;

2. Cycle of $n$-order periodicity: $X^{*}$ is a periodic-point of order $n$ of $X$ if there is a constant $n=2,3, \ldots$ such that $X^{*}=G^{(n)}\left(X^{*}\right)$, where $G^{(n)}(X)$ represents the $n^{\text {th }}$ iterate of $X$;

3. A-periodicity: long-term dynamic outcome of a system for which it is not possible to identify the existence of a fixed-point or of a cycle of a defined periodicity;

4. Chaos: particular form of a-periodicity, which can be defined through the Li and York (1975) theorem - a continuous system $X_{t+1}=G\left(X_{t}\right)$ exhibits chaos if it is possible to identify a periodic point of a period that is not a power of 2 .

Some examples are trivial in this literature. The most frequently addressed are the logistic map and the tent map. They are both defined in one dimension and are good examples of how one evolves from stability to chaos by varying some parameter's value. Their analytical representations are as follows:

- Logistic map:

$$
x_{t+1}=a x_{t}\left(1-x_{t}\right), \quad x_{0} \text { given, } a>1
$$

- Tent map:

$$
x_{t+1}=\left\{\begin{array}{c}
(1 / b) x_{t}, 0 \leq x_{t} \leq b \\
(1 / b)\left(1-x_{t}\right), b<x_{t} \leq 1
\end{array}, \quad x_{0} \text { given, } b \in(0,1)\right.
$$

Figures 1 and 2 present, for each one of the maps, the corresponding long-term trajectories for values of parameters in which the chaotic zone is reached; figure 1 also includes a panel with the bifurcation diagram of the logistic map. The trajectories show that the variables evolve around the corresponding fixed-points, but they will never stabilize in order to rest forever in that specific point. Such behavior is just the result of the shape of the considered nonlinear relation. In the second case, the tent map, we realize that a discontinuity is a possible cause of bounded instability in deterministic dynamics. 

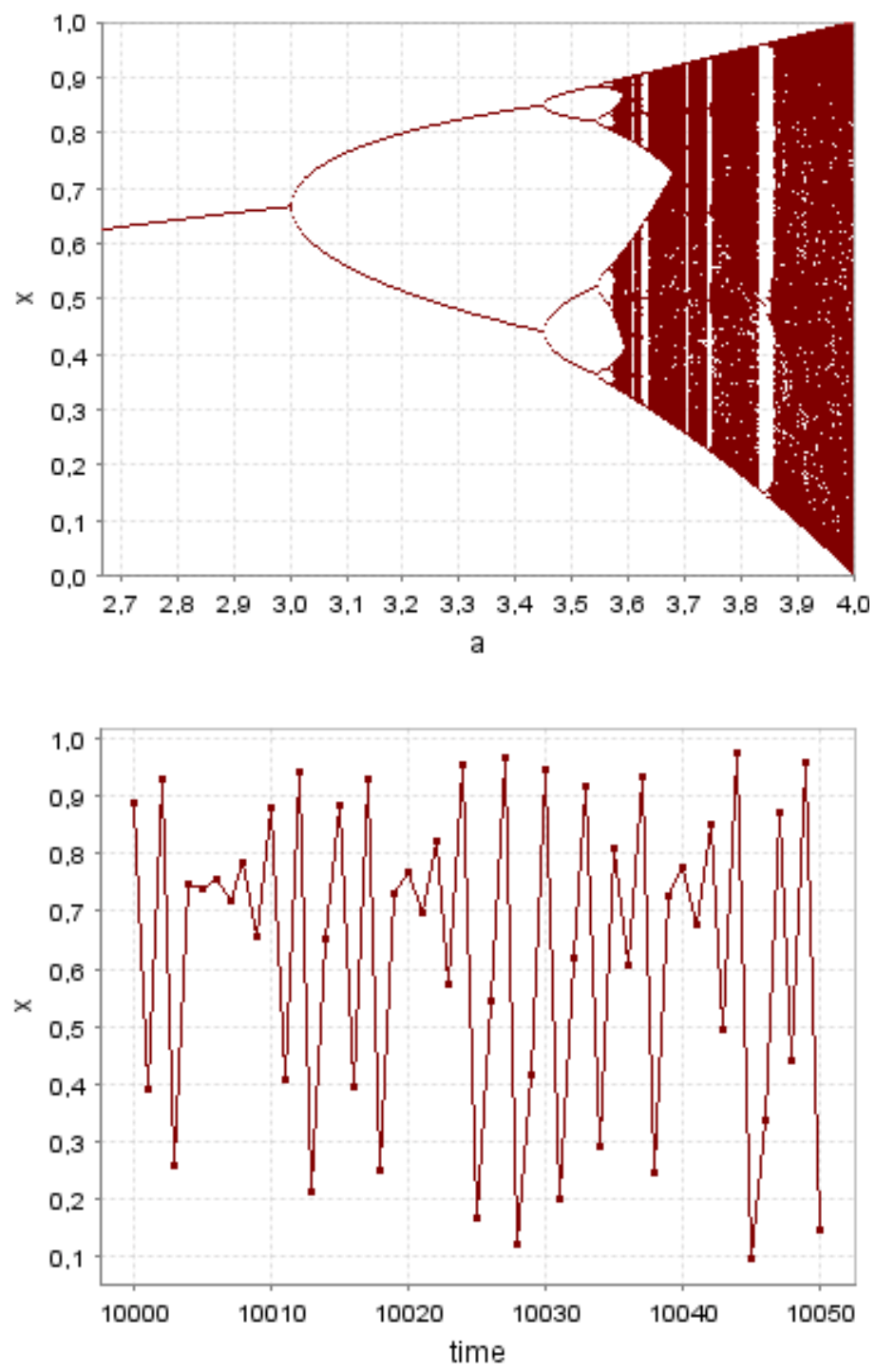

Fig.1 - Logistic map: bifurcation diagram and long-term time series

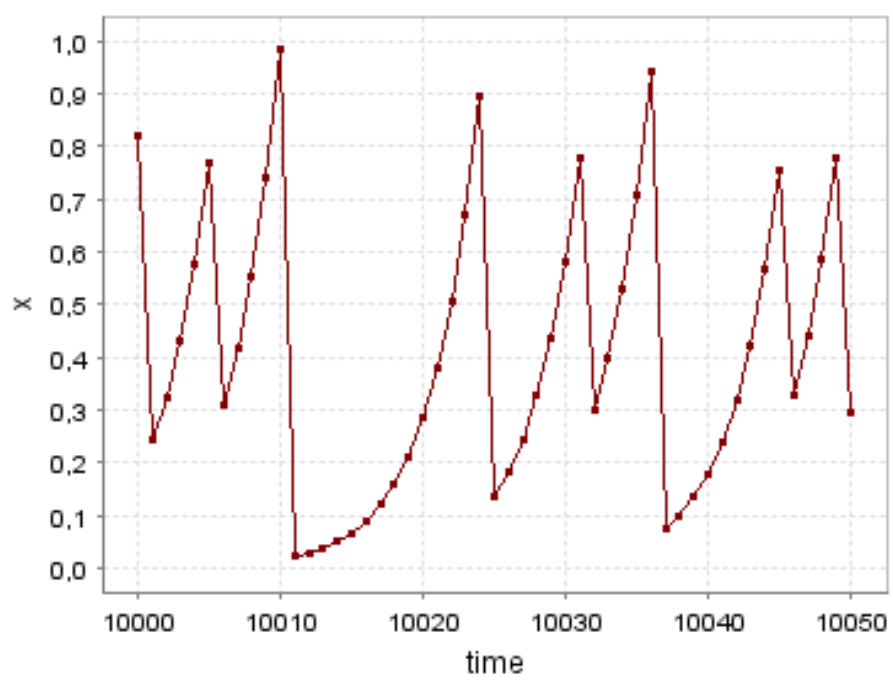

Fig.2 - Tent map: long-term time series 
There are several ways in which we can define nonlinear results and particularly chaos. The developments in nonlinear dynamic theory along the past few decades have allowed to develop important tools to measure chaos in theoretical and empirical terms. It is not the purpose of this paper to deal with these in further detail, but we should stress again the relevance of encountering nonlinear deterministic processes that look random. The property of SDIC implies that, although deterministic, a chaotic system positioned at slightly different states will rapidly evolve towards dramatically different trajectories, which turn forecasting difficult but not impossible. Knowing with accuracy the underlying dynamic process associated with some financial or economic relation and accurately perceiving, as well, the state the system is in, at a precise moment, may allow, at least in the short-run, to predict part of the observed time series volatility.

\section{MAKING SENSE OF THEORETICAL NONLINEARI- TIES}

The usefulness of nonlinear dynamic models in addressing financial and economic phenomena was made clear in the previous sections. Interpreting all observed volatility as 'noise' or 'unexplainable shocks' is a recognition of the incapacity of the researcher in explaining one of the two components of any time series: although the forces shaping long-run trends are relatively easy to address, fluctuations around such trends are typically associated with random events that no theory should ever dare to approach if one wants to keep scientific knowledge as an objective and non-speculative entity.

Although tests exist to measure the possible presence of chaos in observable time series, the results so far are far from conclusive. Tests on chaos for stock prices, interest rates or exchange rates [Barnett and Chen (1988), Serletis and Gogas (1997)] point to a possible affirmative answer, but even if we agree that observed fluctuations are chaotic more than they are random, we will have to face a second challenge: to discern where can we find the source of deterministic fluctuations, i.e., what kind of nonlinear relation effectively exists in order to generate such type of volatility. This is the main question we place in this section - if chaos explains at least partially observed fluctuations, how should we look at economic relations in order to explain the existence of this phenomenon?

The raised question should be approached taking into account the strength of the evidence on chaos, but also under the more philosophical notion, that Ruelle (1994) emphasizes, that 'noise' is only 'noise' as long as we are unable to find some relation that explains it and, thus, a full understanding of how the world works could hypothetically give us a model where all volatility could be endogenously explained.

One of the most influential models in economics and finance involving an interpretation for deterministic randomness is the heterogeneous beliefs model of Brock and Hommes (1997, 1998). In this model, two types of agents are considered: fundamentalists, who are well informed agents that formulate rational expectations, and chartists or trend followers, 
who rely on past information in order to predict future values. These two types of agents will exist both in financial markets and in commodity markets, and the relevant expectations are typically associated to asset prices or prices of goods and services. By combining agents' heterogeneity with a mechanism of discrete choice and evolutionary selection, this kind of framework is able to generate endogenous fluctuations and, therefore, to provide an endogenous explanation for part of the observed volatility.

The mechanism of discrete choice, developed by McFadden (1973), is based on a concept of bounded rationality. Under rational expectations, agents' heterogeneity would not persist; agents with expectations other than the ones involving the fundamental outcome would be expelled from the market because they would be systematically wrong. This notion, when applied to financial markets, is known as the efficient market hypothesis $(\mathrm{EMH})$ : only the fundamental outcome matters, because any expectations other than rational ones imply incurring in systematic mistakes and, thus, lead to an irrational behavior. In this case, markets should be efficient and past prices must not be used to predict future prices. In other words, there is no place in an efficient market for chartists or technical traders; an efficient market is a market of homogeneous and rational traders. A corollary of this reasoning is that an efficient market is also a market where all observed volatility is necessarily exogenous.

Empirical results pointing to phenomena of excess volatility [Shiller (1981)] or to the notion of volatility clustering, already referred in the introduction, indicate that markets are not efficient, rationality may be bounded and technical traders that extrapolate future outcomes from past performance are able to remain on the market without incurring in systematic losses. While fundamentalists believe that prices return to their fundamental value (the discounted sum of future dividends, in the case of asset prices), technical traders exploit particular episodes of more or less strong market activity. This second type of traders works as a destabilizing force, while fundamentalists have the role of stabilizing the market. It is the interplay between these two types of agents that gives rise, at least partially, to the kind of bounded instability price dynamics one observes in practice.

The popularity of the Brock-Hommes framework relates to its capacity of offering a simple but convincing explanation for observed market volatility. This interpretation is associated to the idea that price movements are driven by endogenous laws of motion which can be discovered only if we relax the notion of market efficiency. Heterogeneity of beliefs and bounded rationality (supported on a mechanism of gradual evolutionary selection) seem to be the required ingredients to build a mechanism that is able to support the stylized facts of financial series: excess volatility, volatility clustering, speculative bubbles, crashes and fat tails for the distribution of returns.

In the last few years, the Brock-Hommes framework has been extended in several directions. Some are relatively straightforward, as the work developed by Brock et al. (2005), who generalize the original framework to include many trader types; in this case, the authors introduce the notion of large type limit in order to show that independently of the degree of heterogeneity, an adaptive evolutionary system is capable of generating 
endogenous volatility. Boswijk et al. (2007) resort to the same kind of setting of heterogeneous agents that are boundedly rational; again, the evolutionary selection mechanism is considered and the emphasis is placed on the incentives to change strategy - relative past profits determine investment decisions. Gaunersdorfer et al. (2008) address, as well, the fundamentalist-chartist setup in financial markets; the technical analysis of bifurcations in this paper allows adding some important insights concerning the issue of volatility clustering. Other studies on complex evolutionary systems involving competing boundedly rational trading strategies in financial markets include Manzan and Westerhoff (2007) and Dieci and Westerhoff (2010), who extend the benchmark framework to the foreign exchange market or, more precisely, to the interaction of stock markets of different countries through the exchange rate market. Many other studies follow the referred approach to address price fluctuations.

The presence of chaos in the mentioned type of model is particularly relevant, as highlighted by Wieland and Westerhoff (2005), because if fluctuations are, even partially, chaotic, then chaos control methods can be applied by central authorities in order to reduce observed volatility. Chaos control may be a fundamental tool in order to solve problems of excess volatility in the markets.

Hommes et al. $(2005,2008)$ conduct laboratory experiments to test the empirical plausibility of the fundamentalist-chartist framework. Markets are simulated from a predefined adaptive evolutionary system and results seem to concur with what theory predicts and with what reality shows: bubbles emerge endogenously and, thus, the advanced explanation can be accepted as successful in replicating the stylized facts of financial environments.

The fundamentalist-chartist approach to endogenous fluctuations has been applied also outside the realm of financial markets. Branch and Evans (2007), Branch and McGough (2009) and Lines and Westerhoff (2010) apply the adaptive evolutionary setup to economic relations and, in particular, to macro relations involving the time paths of output and inflation. Volatility clustering is also found in macroeconomic time series (namely, in what concerns the 'Great Moderation' of the 1980s, period in which inflation and output volatility has fallen dramatically). To explain this phenomenon, the cited authors build frameworks where agents either use a sophisticated costly predictor or a simple cheap predictor. Evolutionary competition concerning the performance of these two predictors will, also in this case, determine a scenario of deterministic long-term cycles, in which there will be a systematic change on the shares of agents that choose to remain with one rule or switch to the other rule. Economic time series are also exposed to changes in the intensity of volatility and the same setup used for the financial analysis can have here a decisive role in order to encounter a reasonable explanation for observed behavior.

A brief inspection of the economic literature allows to find many other sources of endogenous volatility that help explaining relevant stylized facts. Some recent examples include: Chen et al. (2008), who apply different types of expectations (perfect foresight, myopic expectations and adaptive expectations) to an overlapping generations model; 
they conclude that dynamics are simple under perfect foresight, but myopic and adaptive expectations may induce cycles and chaotic motion. In Fanti and Manfredi (2007), a standard neoclassical labor market is studied; in this case, cycles and chaos are the result of a setting where consumption and leisure are considered sufficiently low substitutes.

In Hallegatte et al. (2008), a growth model under the absence of market clearing is explored; the authors call it non-equilibrium dynamic model (NEDyM). The NEDyM might generate endogenous business cycles under peculiar conditions. These conditions involve two types of inertia: delays in the mutual adjustment between production and demand and a delayed dependence of investment on past profits. Thus, part of the observed volatility associated to business cycles can, in this perspective, be associated with these two inertia effects. Chaotic motion is also found and explored by Sushko et al. (2010) in a growth setting where investment decisions are central: investment will be delimited from above and from below given capacity limits and capital depreciation, respectively. The ceiling and the floor are ingredients that are likely to generate cycles and chaos for reasonable combinations of parameter values. Many other studies, involving different types of explanations explore endogenous fluctuations in macroeconomic environments. Just to give two additional references, we cite the work of Yokoo and Ishida (2008), who find an explanation for endogenous business cycles on deficiencies on the access to and interpretation of relevant information (what they call misperceptions), and Kikuchi and Stachurski (2009), who study international growth and attribute fluctuations to the interaction through credit markets when countries have asymmetric economic conditions.

\section{AN ILLUSTRATION: INFLATION DYNAMICS}

In this section, we present our own illustration on how endogenous volatility might arise once we take some acceptable changes over a benchmark macroeconomic model.

We follow Mankiw and Reis (2002) and consider a monopolistically competitive market environment, where firms want to set an optimal or desired price, that is obtained by taking a trivial profit maximization problem. We define the desired price as $p_{t}^{*}$; the aggregate price level is given by $p_{t}$ and $y_{t}$ represents the output gap (the relation between effective and potential output). All these variables are expressed in logarithms of the corresponding original values. The firms' optimization problem leads to the desired price

$$
p_{t}^{*}=p_{t}+\alpha y_{t}
$$

with $\alpha \in(0,1)$ a measure of real rigidities (this parameter translates the degree of substitutability between different varieties of the assumed good; $\alpha=0$ brings us back to perfect competition). The equation states that the price firms intend to set at some period $t$ will be larger than the observed price level in periods of expansion $\left(y_{t}>0\right)$ and smaller than the observed price level in periods of recession $\left(y_{t}<0\right)$.

We will assume that a share $\lambda$ of firms collects information on the state of the economy 
at the current period, while the remaining share $1-\lambda$ resorts to old information, obtained one period in the past. Thus, the observed price level will be

$$
p_{t}=\lambda p_{t}^{0}+(1-\lambda) p_{t}^{1}
$$

with $p_{t}^{0}=p_{t}^{*}$ and $p_{t}^{1}=E_{t-1}\left(p_{t}^{*}\right)$. We also assume that if expectations are formed at $t-1$, firms will perceive the current period as the long-run steady-state and the expectation will correspond to the observed desired price at $t-1$ plus the rate of growth to $t$; as it will become clear at a later stage, the growth rate of the output gap will be zero in the steady-state and inflation will grow at some rate $\pi^{*}$ that we will be able to present in explicit form. The expectation is, then, $E_{t-1}\left(p_{t}^{*}\right)=p_{t-1}+\pi^{*}+\alpha y_{t-1}$.

We define the inflation rate as $\pi_{t}:=p_{t}-p_{t-1}$. Putting together the previous information, we arrive to a supply side relation or Phillips curve that establishes a link between the output gap and the inflation rate:

$$
\pi_{t}=\frac{\alpha \lambda}{1-\lambda} y_{t}+\alpha y_{t-1}+\pi^{*}
$$

On the demand side of the economy, we will have to take a trivial utility maximization problem. We also consider information stickiness on the part of households. As for firms, stickiness will translate on a share $\lambda$ of consumers that set their consumption plans at $t$ and a share $1-\lambda$ that has updated their information set at $t-1$. Thus,

$$
c_{t}=\lambda c_{t, 0}+(1-\lambda) c_{t, 1}
$$

Variable $c_{t}$ represents the logarithm of aggregate consumption and $c_{t, 0}, c_{t, 1}$ are, respectively, the consumption levels (in logs) of each one of the two types of assumed households. According to Mankiw and Reis (2006), $c_{t, j}=-\theta E_{t-j}\left(R_{t}\right), j=0,1$, with $\theta>0$ the intertemporal elasticity of substitution of consumption and $R_{t}=E_{t}\left(\sum_{i=0}^{\infty} r_{t+i}\right)$ the long real interest rate. If the real interest rate, $r_{t}$, is expected to converge to its long-run value, which is zero, at a rate $a \in(0,1)$, then, we can simplify the expression: $R_{t}=\sum_{i=0}^{\infty}(1-a)^{i} r_{t}=\frac{1}{a} r_{t}$.

Assuming market clearing, i.e., $y_{t}=c_{t}$, expression (4) will be equivalent to:

$$
y_{t}=-\frac{\theta}{a}\left[\lambda r_{t}+(1-\lambda) r_{t-1}\right]
$$

Equation (5) can be further rearranged by taking the Fisher equation, $i_{t}=r_{t}+$ $E_{t}\left(\pi_{t+1}\right)$, where $i_{t}$ stands for the nominal interest rate. We must consider as well a monetary policy rule; here, the assumption is that the central bank reacts to deviations of the expected inflation rate relatively to a target value $\bar{\pi}$, when setting the nominal interest rate,

$$
i_{t}=\phi\left[E_{t}\left(\pi_{t+1}\right)-\bar{\pi}\right]
$$

The monetary policy is considered active, in the sense that the monetary authority re- 
sponds aggressively to changes on the inflation rate, that is, $\phi>1$.

Assuming households exhibit perfect foresight when predicting future inflation, the demand side equation takes the form,

$$
y_{t}=-\frac{\theta(\phi-1)}{a}\left[\lambda \pi_{t+1}+(1-\lambda) \pi_{t}-\pi^{*}\right]
$$

The behavior of this economy is fully described by the supply and demand relations (3) and (7). Combining the two, we can suppress the output-gap from the analysis and arrive to a system that explains the evolution of the inflation rate in time. This system is:

$$
\left\{\begin{array}{l}
\pi_{t+1}=-\left[\frac{a(1-\lambda)}{\alpha \lambda^{2} \theta(\phi-1)}+\frac{2(1-\lambda)}{\lambda}\right]\left(\pi_{t}-\pi^{*}\right)-\left(\frac{1-\lambda}{\lambda}\right)^{2}\left(z_{t}-\pi^{*}\right)+\pi^{*} \\
z_{t+1}=\pi_{t}
\end{array}\right.
$$

with $\pi^{*}=\frac{\phi}{\phi-1} \bar{\pi}$ the steady-state inflation level. Note that the steady-state inflation rate is larger than the target that is set by the central bank; this is the result of considering a non-optimal monetary policy rule.

The dynamics of system (8) can be addressed under a local perspective, i.e., in the vicinity of the steady-state and under a global analysis. The first typically allows for separating regions of stability and instability; the global analysis confirms the location of the stability area and allows to perceive if the region of instability involves some kind of cyclical motion.

To address the model's dynamics, we consider an additional assumption: we assume that $\lambda$ is not constant; this degree of attentiveness will respond to the inflation observed in the previous period, i.e., $\lambda=\lambda\left(z_{t}\right)$. The idea is that agents will be more attentive if they have previously observed a larger inflation level; low levels of inflation would not require such an attentive behavior. Thus, $\lambda$ will be an increasing function of $z_{t}$. If we define a floor $\underline{\lambda} \in(0,1)$ that corresponds to the lowest possible level of attention of firms and households and an attentiveness share $\lambda_{0} \in(\underline{\lambda}, 1)$ such that $\lambda_{0}=\lambda(0)$, the function that translates the behavior of the degree of attentiveness will be something as presented in figure 3, where large levels of past inflation imply full attentiveness (i.e., all agents will be attentive to relevant news); in other words, as $z_{t} \rightarrow+\infty, \lambda$ converges asymptotically to 1 . 


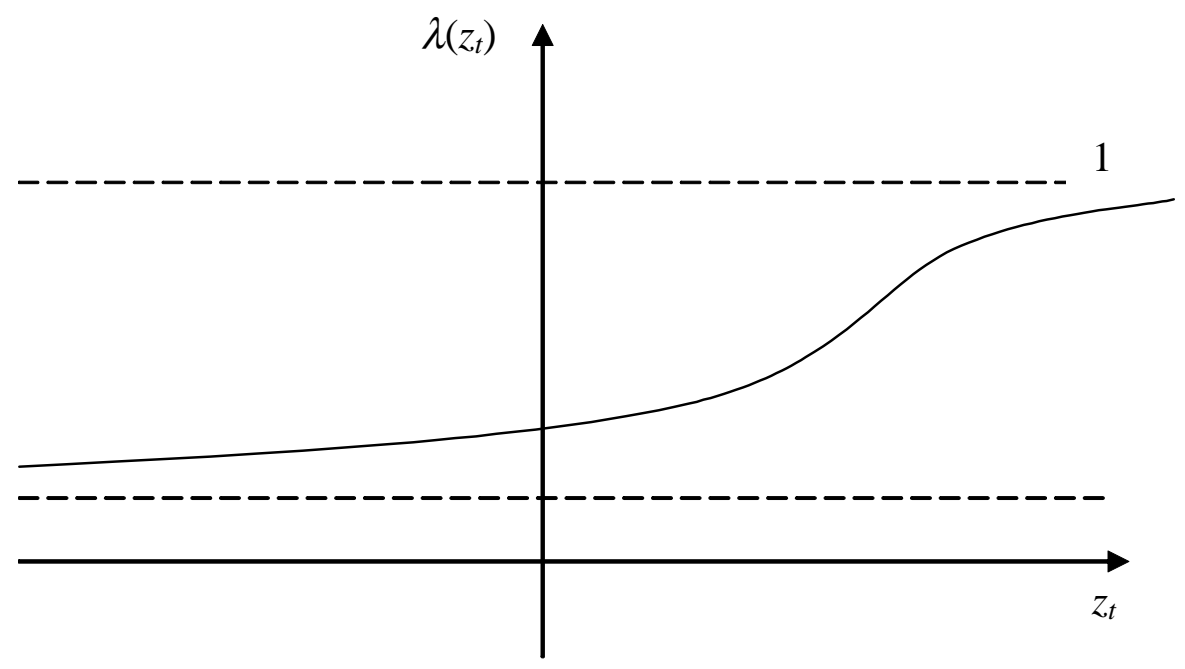

Fig. $3-\lambda\left(z_{t}\right)$ function

The function in figure 3 can be analytically represented in the following form: ${ }^{1}$

$$
\lambda\left(z_{t}\right)=\frac{1+\underline{\lambda}}{2}-\frac{1-\underline{\lambda}}{\pi} \arctan \left[\tan \left(\frac{\pi}{2} \frac{1+\underline{\lambda}-2 \lambda_{0}}{1-\underline{\lambda}}\right)-z_{t}\right]
$$

Local dynamics are similar with a constant and with an increasing degree of attentiveness. The linearized system is:

$$
\left[\begin{array}{c}
\pi_{t+1}-\pi^{*} \\
z_{t+1}-\pi^{*}
\end{array}\right]=\left[\begin{array}{cc}
-\left[\frac{a\left(1-\lambda^{*}\right)}{\alpha \lambda^{* 2} \theta(\phi-1)}+\frac{2\left(1-\lambda^{*}\right)}{\lambda^{*}}\right] & -\left(\frac{1-\lambda^{*}}{\lambda^{*}}\right)^{2} \\
0
\end{array}\right]\left[\begin{array}{c}
\pi_{t}-\pi^{*} \\
z_{t}-\pi^{*}
\end{array}\right]
$$

with $\lambda^{*}$ the steady-state level of $\lambda\left(z_{t}\right)$. Applying stability conditions $1-\operatorname{Det}(J)>0$, $1-\operatorname{Tr}(J)+\operatorname{Det}(J)>0$ and $1+\operatorname{Tr}(J)+\operatorname{Det}(J)>0$, with $J$ the Jacobian matrix of the above system, we conclude that only the second condition is universally satisfied. The first and the third conditions imply the following inequalities, respectively,

$$
\lambda^{*}>1 / 2 ; \quad \phi>1+\frac{a\left(1-\lambda^{*}\right)}{\alpha \theta\left[1-4 \lambda^{*}\left(1-\lambda^{*}\right)\right]}
$$

That is, stability requires a relatively high degree of attentiveness and, simultaneously, a relatively aggressive monetary policy.

Through a global dynamic analysis we can confirm that the region of stability is bounded according to the found inequalities and that endogenous volatility exists for specific combinations of parameter values. Take the following set of reasonable values: $a=0.01, \alpha=0.1, \theta=1, \underline{\lambda}=0.1, \bar{\pi}=0.02$ and $\phi=1.5$. Figure 4 presents a bifurcation diagram that allows to perceive that different values of $\lambda_{0}$ generate qualitatively different outcomes in terms of dynamics. Chaotic motion exists for specific values of the referred parameter; for instance, for $\lambda_{0}=0.538$ there is chaos, as one observes by looking at the strange attractor in figure 5 and to the long-run time series of inflation in figure 6 .

\footnotetext{
${ }^{1}$ Note that the $\pi$ in the expression is not the inflation rate but the value $3.14159 \ldots$
} 


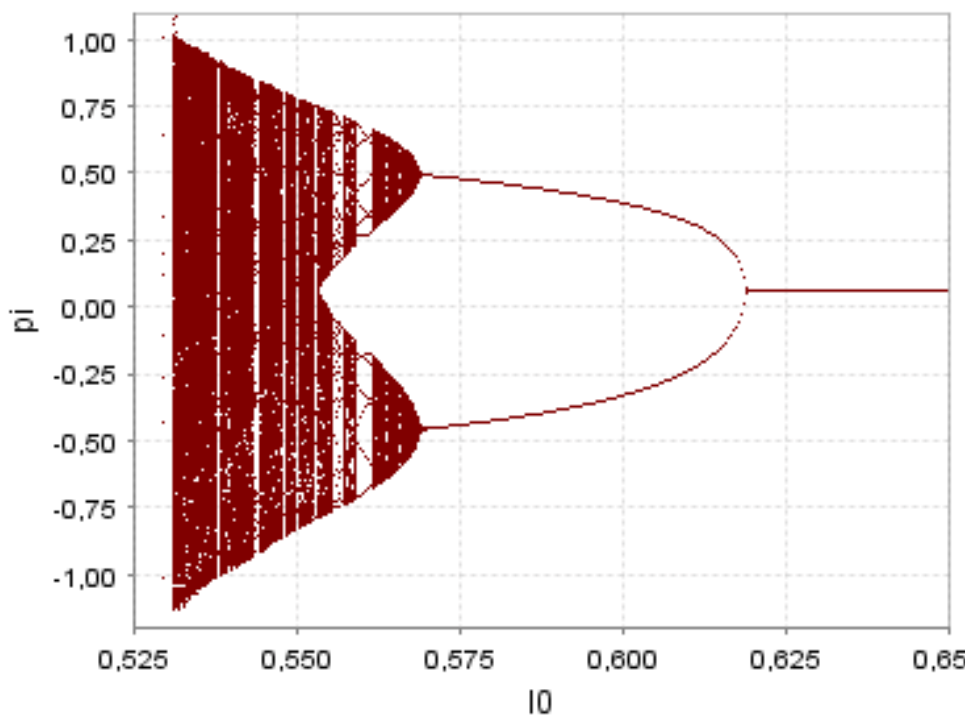

Fig.4 - Bifurcation diagram $\left(\pi_{t} ; \lambda_{0}\right)$

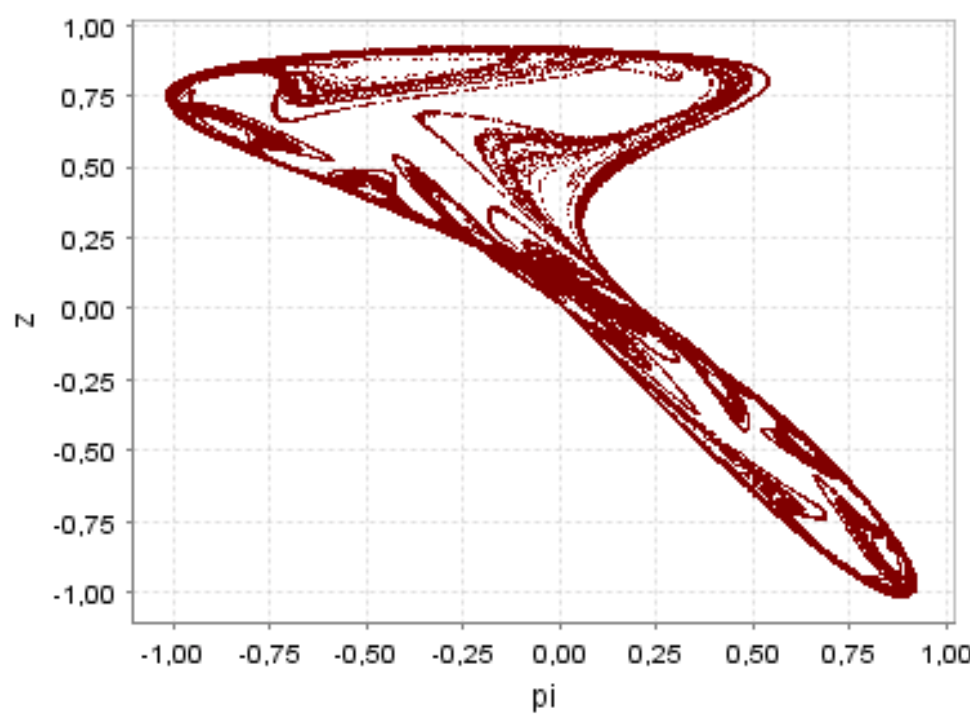

Fig.5 - Chaotic attractor $\left(\pi_{t}, z_{t}\right) ; \lambda_{0}=0.538$ 


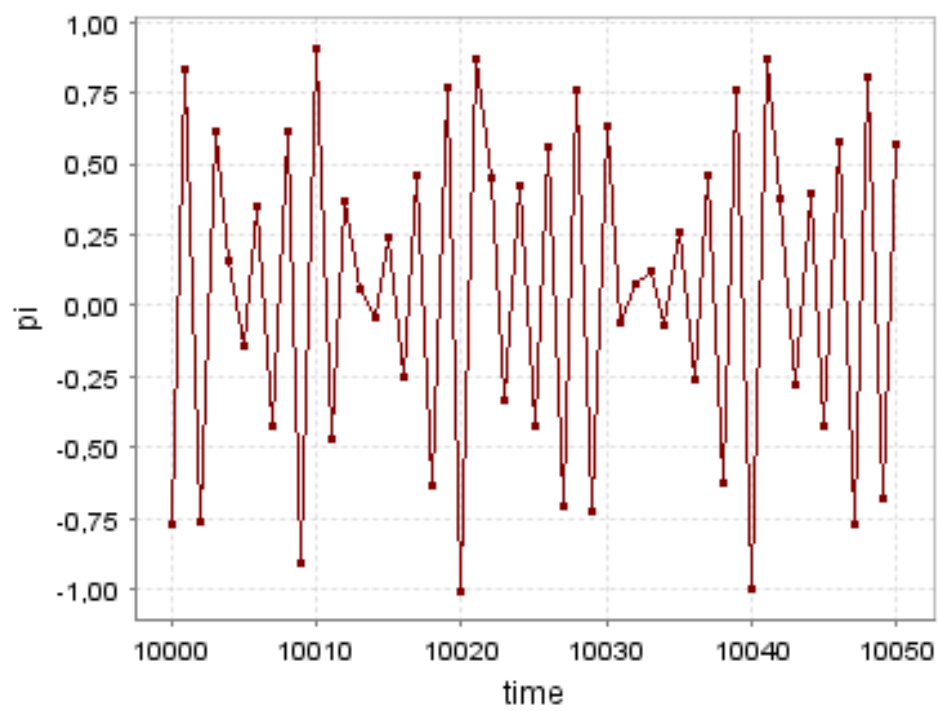

Fig.6 - Long-term time trajectory of $\pi_{t} ; \lambda_{0}=0.538$

The above illustration shows how a conventional benchmark macroeconomic model, that takes as structural elements a Phillips curve and an aggregate demand equation, can be slightly modified in order to provide for the existence of endogenous volatility: in this case, the volatility is simply the result of how one approaches attentiveness relatively to the relevant information.

\section{CONCLUSION}

Time series of economic and financial variables display volatility and this volatility presents features that indicate that fluctuations do not correspond to pure noise. Being able to identify the reasons why volatility assumes some specific patterns may be helpful in understanding aggregate phenomena. A popular interpretation, namely in finance, considers a setting of heterogeneous boundedly rational agents. The interaction between fundamentalists and chartists in an evolutionary setting tends to generate endogenous fluctuations. Many other candidates to justify deterministic volatility exist, as discussed in the paper.

We have presented our own illustration, where varying degrees of attentiveness trigger the formation of endogenous cycles for the inflation rate (and also for the output gap, because these two variables are correlated). The main lesson we withdraw is that endogenous volatility is a frequently obtainable result in any dynamic system involving nonlinearities. If we have the possibility of translating complex observable phenomena into nonlinear mathematical relations, we might be able to find convincing explanations on why observed fluctuations are not completely stochastic. 


\section{REFERENCES}

- Adrangi, B.; M. Allender; A. Chatrath and K. Raffiee (2010). "Nonlinearities and Chaos: Evidence from Exchange Rates." Atlantic Economic Journal, vol. 38, pp. $247-248$.

- Barnett, W.A. and P. Chen (1988). "The Aggregation-Theoretic Monetary Aggregates are Chaotic and Have Strange Attractors: an Econometric Application of Mathematical Chaos." In W. A. Barnett, E. Berndt and H. White (eds.), Dynamic Econometric Modelling, Cambridge: Cambridge University Press, pp. 199-245.

- Barnett, W.A.; A. Serletis and D. Serletis (2006). "Nonlinear and Complex Dynamics in Real Systems." International Journal of Nonlinear Sciences and Numerical Simulation, vol. 7, pp. 191-196.

- Bollerslev, T. (1986). "Generalized Autoregressive Conditional Heteroscedasticity." Journal of Econometrics, vol. 31, pp. 307-327.

- Boswijk, H.P.; C.H. Hommes and S. Manzan (2007). "Behavioral Heterogeneity in Stock Prices." Journal of Economic Dynamics and Control, vol. 31, pp. 1938-1970.

- Branch, W.A. and G.W. Evans (2007). "Model Uncertainty and Endogenous Volatility." Review of Economic Dynamics, vol. 10, pp. 207-237.

- Branch, W.A. and B. McGough (2009). "A New-Keynesian Model with Heterogeneous Expectations." Journal of Economic Dynamics and Control, vol. 33, pp. $1036-1051$.

- Brock, W.A. and C.H. Hommes (1997). "A Rational Route to Randomness." Econometrica, vol. 65, pp.1059-1095.

- Brock, W.A. and C.H. Hommes (1998). "Heterogeneous Beliefs and Routes to Chaos in a Simple Asset Pricing Model." Journal of Economic Dynamics and Control, vol. 22 , pp. $1235-1274$.

- Brock, W.A.; C.H. Hommes and F.O.O. Wagener (2005). "Evolutionary Dynamics in Markets with Many Trader Types." Journal of Mathematical Economics, vol. 41, pp. $7-42$.

- Chen, H-J.; M-C Li and Y-J Lin (2008). "Chaotic Dynamics in an Overlapping Generations Model with Myopic and Adaptive Expectations." Journal of Economic Behavior and Organization, vol. 67, pp. 48-56.

- Dieci, R. and F. Westerhoff (2010). "Heterogeneous Speculators, Endogenous Fluctuations and Interacting Markets: a Model of Stock Prices and Exchange Rates." Journal of Economic Dynamics and Control, vol. 34, pp. 743-764. 
- Engle, R.F. (1982). "Autoregressive Conditional Heteroscedasticity with Estimates of the Variance of United Kingdom Inflation." Econometrica, vol. 50, pp. 987-1007.

- Fanti, L. and P. Manfredi (2007). "Neoclassical Labour Market Dynamics, Chaos and the Real Wage Phillips Curve." Journal of Economic Behavior and Organization, vol. 62 , pp. 470-483.

- Gaunersdorfer, A.; C.H. Hommes and F.O.O. Wagener (2008). "Bifurcation Routes to Volatility Clustering under Evolutionary Learning." Journal of Economic Behavior and Organization, vol. 67, pp. 27-47.

- Gomes, O. (2006). "Routes to Chaos in Macroeconomic Theory." Journal of Economic Studies, vol. 33, pp. 437-468.

- Grandmont, J.-M. (2008). "Nonlinear Difference Equations, Bifurcations and Chaos: an Introduction." Research in Economics, vol. 62, pp. 122-177.

- Hallegatte, S.; M. Ghil; P. Dumas and J.C. Hourcade (2008). "Business Cycles, Bifurcations and Chaos in a Neo-Classical Model with Investment Dynamics." Journal of Economic Behavior and Organization, vol. 67, pp. 57-77.

- Hommes, C.H.; J. Sonnemans; J. Tuinstra and H. van de Velden (2005). "A Strategy Experiment in Dynamic Asset Pricing." Journal of Economic Dynamics and Control, vol. 29 , pp. 823-843.

- Hommes, C.H.; J. Sonnemans; J. Tuinstra and H. van de Velden (2008). "Expectations and Bubbles in Asset Pricing Experiments." Journal of Economic Behavior and Organization, vol. 67, pp. 116-133.

- Kikuchi, T. and J. Stachurski (2009). "Endogenous Inequality and Fluctuations in a Two-Country Model." Journal of Economic Theory, vol. 144, pp. 1560-1571.

- Li, T. and J. Yorke (1975). "Period Three Implies Chaos." American Mathematical Monthly, vol. 82, pp. 985-992.

- Lines, M. (Ed.) (2005). Nonlinear Dynamical Systems in Economics. New York: Springer.

- Lines, M. and F. Westerhoff (2010). "Inflation Expectations and Macroeconomic Dynamics: the Case of Rational versus Extrapolative Expectations." Journal of Economic Dynamics and Control, vol. 34, pp. 246-257.

- Mandelbrot, B. (1963). "The Variation of Certain Speculative Prices." Journal of Business, vol. 36, pp. 394-419.

- Mankiw, N.G. and R. Reis (2002). "Sticky Information versus Sticky Prices: a Proposal to Replace the New Keynesian Phillips Curve." Quarterly Journal of Economics, vol. 117, pp. 1295-1328. 
- Mankiw, N.G. and R. Reis (2006). "Pervasive Stickiness." American Economic Review, vol. 96, pp. 164-169.

- Manzan, S. and F. Westerhoff (2007). "Heterogeneous Expectations, Exchange Rate Dynamics and Predictability." Journal of Economic Behavior and Organization, vol. 64, pp. 111-128.

- McFadden, D. (1973). "Conditional Logit Analysis of Qualitative Choice Behavior." In P. Zarembka (ed.), Frontiers in Econometrics. New York: Academic Press, pp.105-142.

- Medio, A. and M. Lines (2001). Nonlinear Dynamics: a Primer. Cambridge, UK: Cambridge University Press.

- Ruelle, D. (1994). "Where Can One Hope to Profitably Apply the Ideas of Chaos?" Physics Today, vol. 47, pp. 24-30.

- Serletis, A. and P. Gogas (1997). "Chaos in East European Black Market Exchange Rates." Research in Economics, vol. 51, pp. 359-385.

- Shiller, R.J. (1981). "Do Stock Prices Move Too Much to be Justified by Subsequent Changes in Dividends?" American Economic Review, vol. 71, pp. 421-436.

- Sushko, I.; L. Gardini and T. Puu (2010). "Regular and Chaotic Growth in a Hicksian Floor/Ceiling Model." Journal of Economic Behavior and Organization, vol. 75 , pp. $77-94$

- Wieland, C. and F. Westerhoff (2005). "Exchange Rate Dynamics, Central Bank Interventions and Chaos Control Methods." Journal of Economic Behavior and Organization, vol. 58, pp. 117-132.

- Yokoo, A. and J. Ishida (2008). "Misperception-driven Chaos: Theory and Policy Implications." Journal of Economic Dynamics and Control, vol. 32, pp. 1732-1753. 